\title{
Protée
}

\section{Suzanne Tardif-Berberi}

Par delà l'empreinte

\section{François Ouellet}

Volume 33, numéro 1, printemps 2005

L’allégorie visuelle

URI : https://id.erudit.org/iderudit/012267ar

DOI : https://doi.org/10.7202/012267ar

Aller au sommaire du numéro

\section{Éditeur(s)}

Département des arts et lettres - Université du Québec à Chicoutimi

ISSN

0300-3523 (imprimé)

1708-2307 (numérique)

Découvrir la revue

Citer cet article

Ouellet, F. (2005). Suzanne Tardif-Berberi : par delà l'empreinte. Protée, 33(1),

58-66. https://doi.org/10.7202/012267ar d'utilisation que vous pouvez consulter en ligne.

https://apropos.erudit.org/fr/usagers/politique-dutilisation/ 


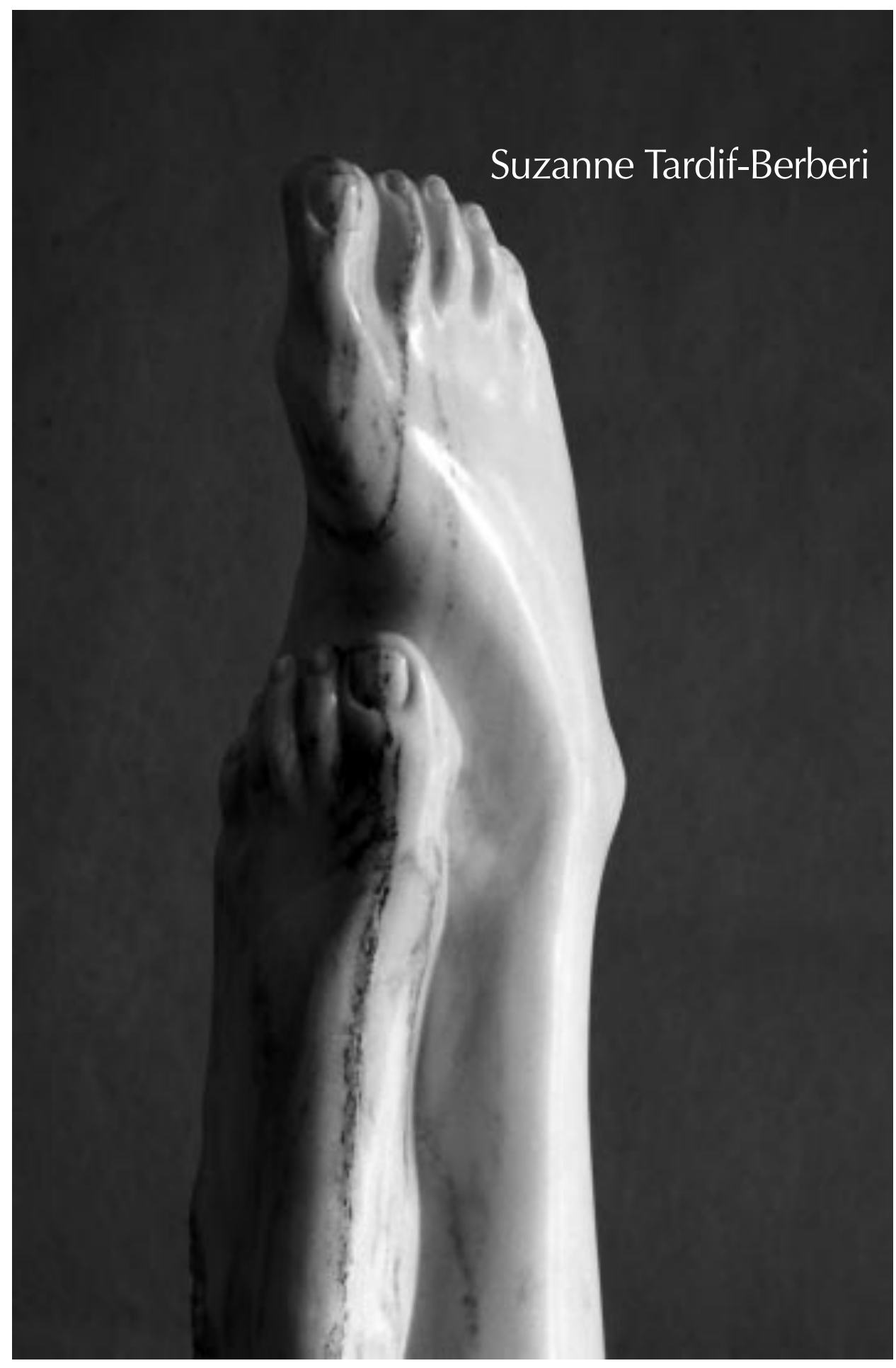

Aphrodite (détail), 1999. Marbre du Vermont, 150 × 30 × $18 \mathrm{~cm}$. 


\section{Par delà l'empreinte}

Depuis quelques années, la sculpteure Suzanne Tardif-Berberi a orienté sa démarche créatrice vers la représentation du pied. Les formes sont belles, dignes, épurées. Mais, de façon délibérée, il ne s'agit surtout pas que d'un marbre. Le marbre est représentation: au-delà du pied sculpté, il y a un symbole; et par delà l'empreinte, il y a un signifiant. « L'empreinte du pied de Vendredi que Robinson découvre au cours de sa promenade dans l'île n'est pas un signifiant. En revanche, à supposer que lui, Robinson, pour une raison quelconque, efface cette trace, là s'introduit nettement la dimension du signifiant », comme le dit Lacan. C'est que le signifiant " témoigne d'une présence passée » ${ }^{1}$, il se donne comme tel dans ce qui est en dessous, il se love en creux. C'est par ce qu'elle recouvre que la trace fait sens et qu'elle institue une quête. Comme c'est par ce qu'elle manifeste que la sculpture recouvre du sens.

Aussi est-ce dans le discours mythologique que puise d'abord Suzanne Tardif-Berberi, car la littérature a créé le mythe comme le mythe a engendré la littérature. La littérature en premier n'a jamais cessé d'écrire la trace, mais aussi de la thématiser, donc de se faire trace de sa propre trace, comme pour mieux faire voir l'espace qu'ouvre le signe:

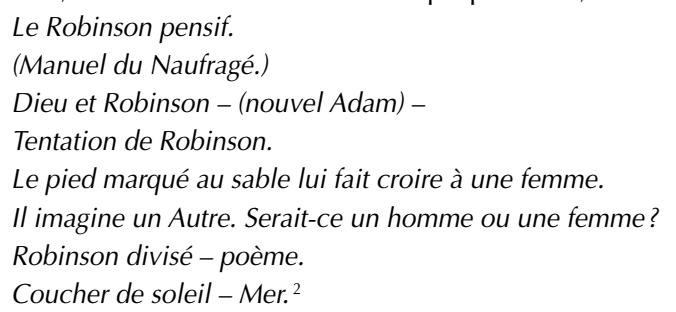

C'est une démarche sculpturale comparable à celle du poète qui inspire le travail de Suzanne Tardif-Berberi, où le pied, par le biais des figures mythologiques, s'énonce, à sa manière particulière, à la fois comme métonymie du désir et comme emblème. Précisément, les œuvres de la sculpteure sont exemplaires en ce qu'elles nous rappellent à la lettre la valeur signifiante et structurante que contient la trace, elles nous indiquent conséquemment la volonté de sens mise en jeu dans I'art. Pierre Vadeboncœur file la métaphore:

Chaque bribe d'existence qu'elle [l'œuvre] isole est comme une pierre où fermement poser le pied de l'absolu et de la pérennité. II n'y a que sables mouvants, mais dans l'art il suffit d'y marcher pour que chaque pas y trouve un point d'appui. Ainsi, l'art emprunte sans cesse à la vie ses réalités décevantes, dont il fait et ne peut faire que des figures de ce qui ne l'est pas. On croirait que c'est à dessein qu'il les prend, pour en changer radicalement le sens. Il s'empare de tout ce qui tombe, pour lui faire tout supporter. ${ }^{3}$

Le travail de Suzanne Tardif-Berberi participe à la fois d'une recherche mythologique et d'une quête métaphysique. Ici, c'est "Héphaïstos le boiteux», handicapé parce que sa mère le jeta du haut de l'Olympe; "Aphrodite», qui accepta de céder à Hermès afin de récupérer la sandale qu'il lui avait volée ; "Hermès au pied léger » ou encore "Le pied nu de Jason». Là, c'est "Les mortels sont passés», une œuvre qui rappelle notre passage sur terre, ou encore "L'axe sacré », un monolithe sur les croyances, rites et symboles de l'Antiquité enfouis dans notre mémoire collective. Cette dernière œuvre, qui montre un pied édifié sur une colonne, célèbre « les pèlerins en marche vers des lieux qui exercent toujours des attraits d'ordre spirituel, écrit l'artiste: Stonehenge, Carnac, Saint-Jacques de Compostelle, La Mecque», etc. Empreinte royale de la vie humaine sur terre, dépositaire de toutes les histoires et de ses signes, ce pied magnifiquement sculpté propose ainsi l'orientation d'un savoir et d'un parcours, d'une traversée dans le temps et dans l'espace.

François Ouellet

1. J. Lacan, Le Séminaire V. Les formations de l'inconscient, Paris, Seuil, 1998, p. 343.

2. P. Valéry, "Robinson», dans La Jeune Parque et poèmes en prose, Paris, Gallimard, coll. "Poésie/Gallimard», 1992 , p. 67.

3. P. Vadeboncœur, Les Deux Royaumes, Montréal, L'Hexagone, 1978, p. 70-71. 


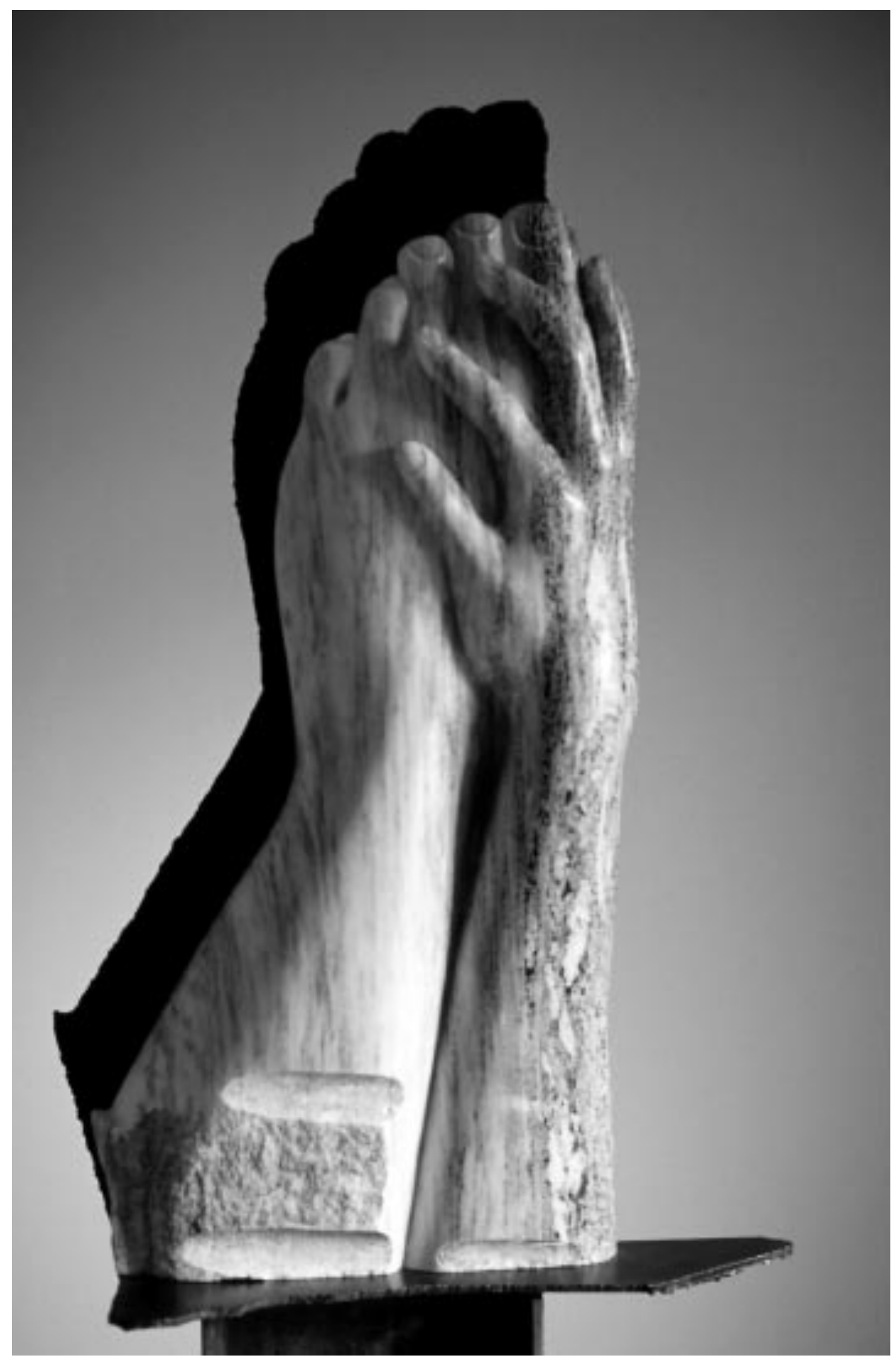

Le retour d'Ulysse (détail), 2001. Marbre du Vermont, 153 x 53 x 36cm. 


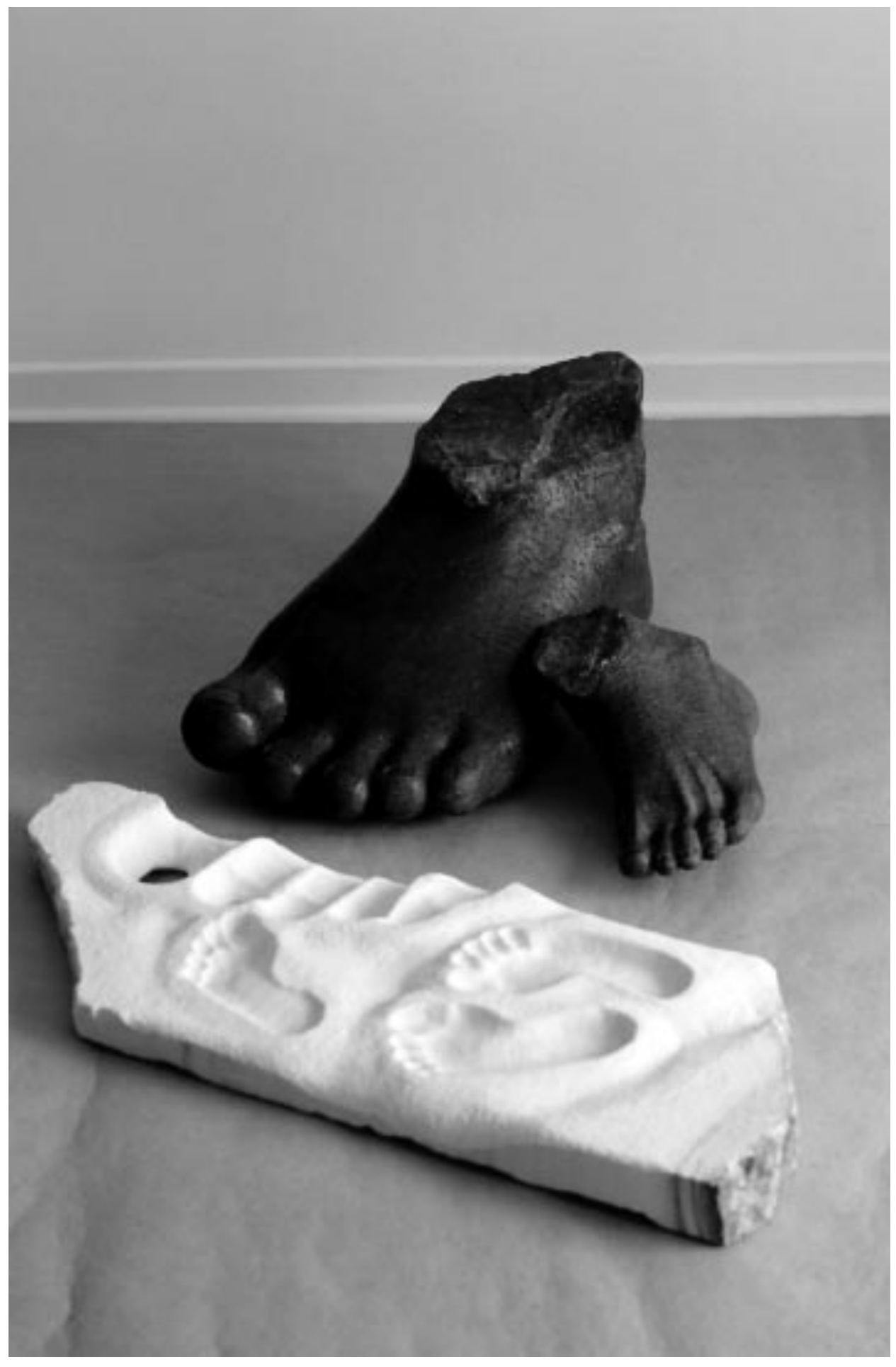

Les mortels sont passés, 2000. Marbre du Vermont et pierre de Saint-Marc, 96 × 38 × 31cm et $94 \times 36 \times 7 \mathrm{~cm}$. 


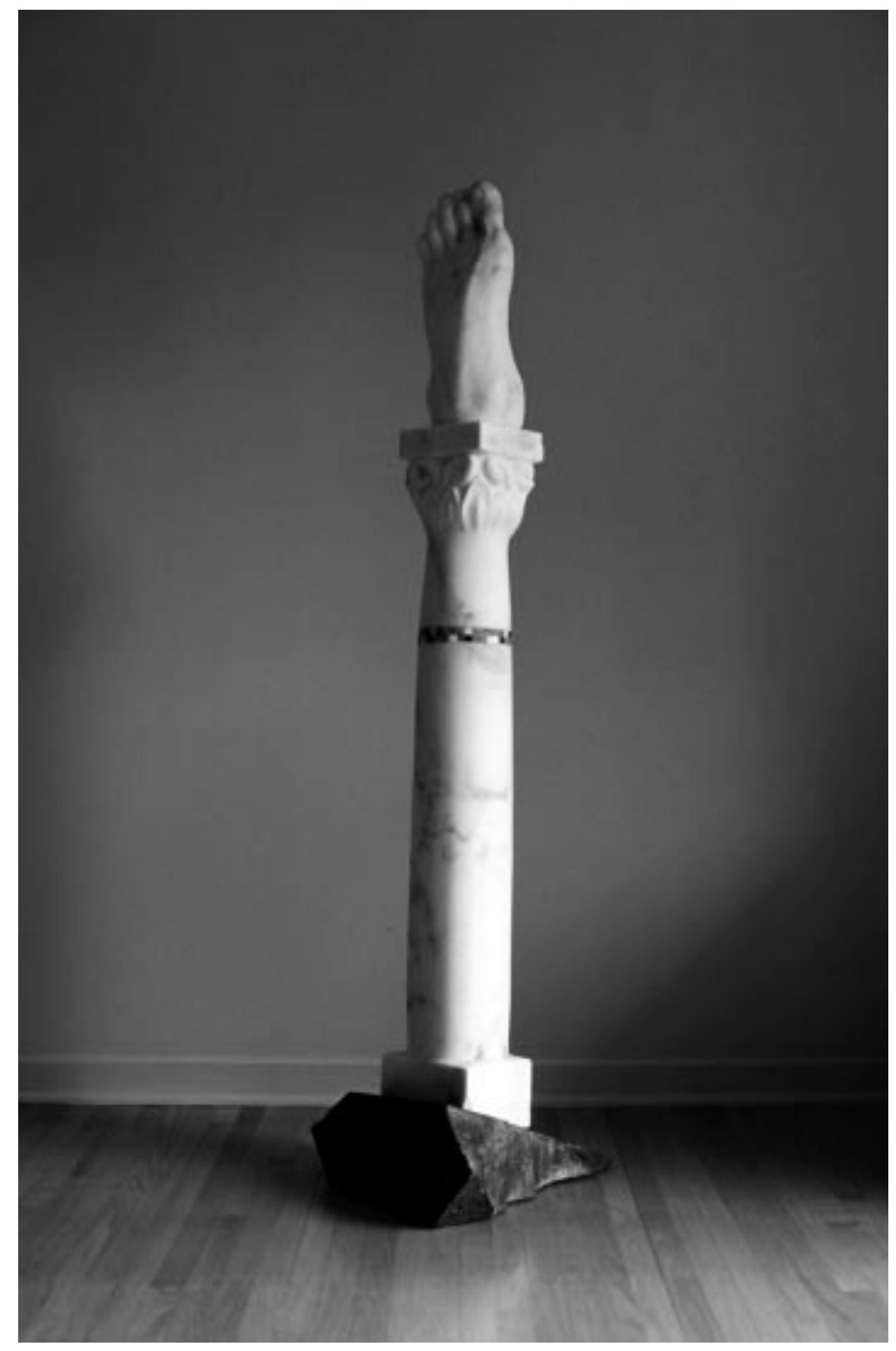

L'axe sacrée (2), 2001. Marbre du Vermont, granit, mosaïque, 160 × 50 × 28cm. 


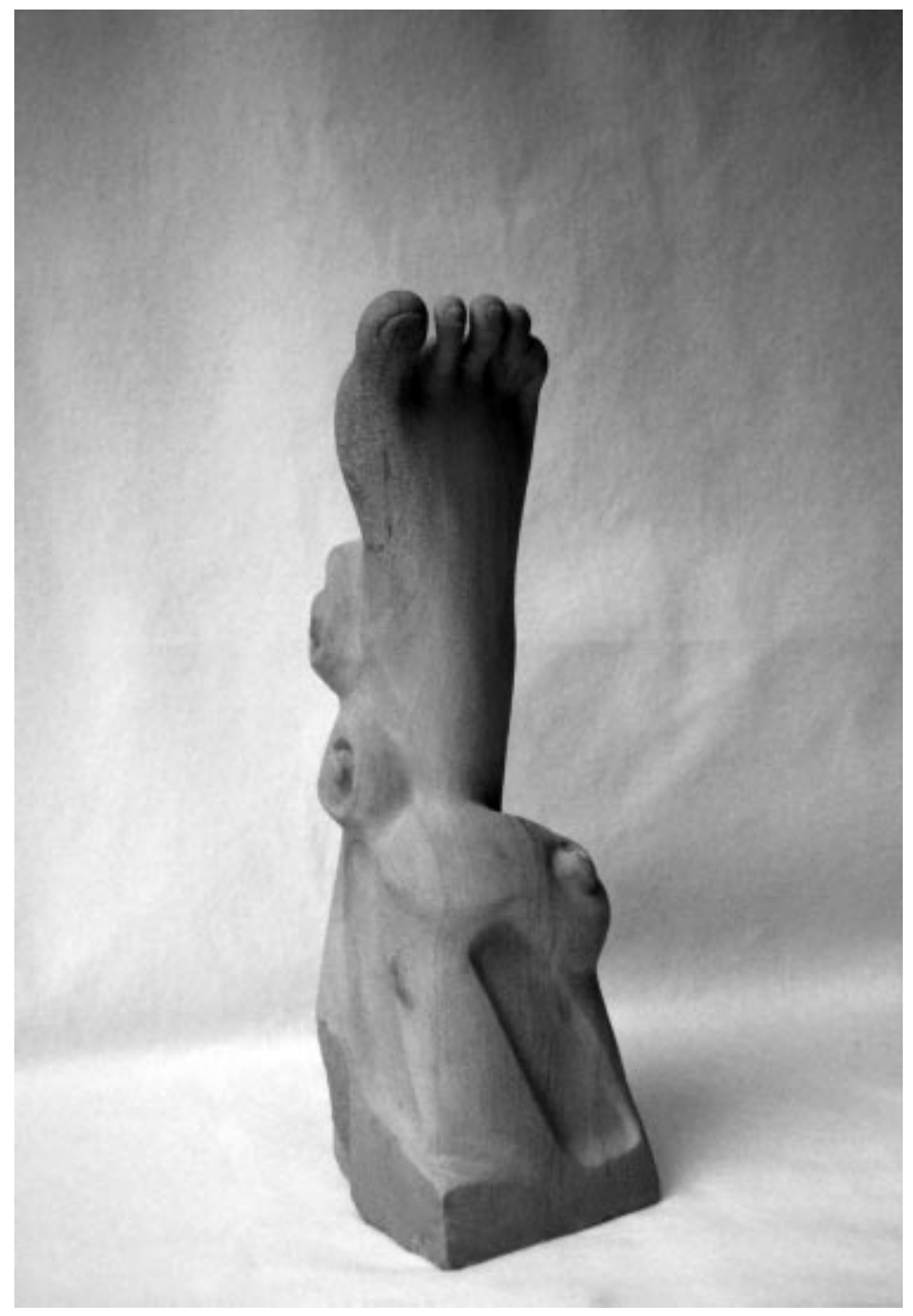

Thésée (piédestal), 2003. Pierre d'Indiana, 59 × 19 × 17cm. 


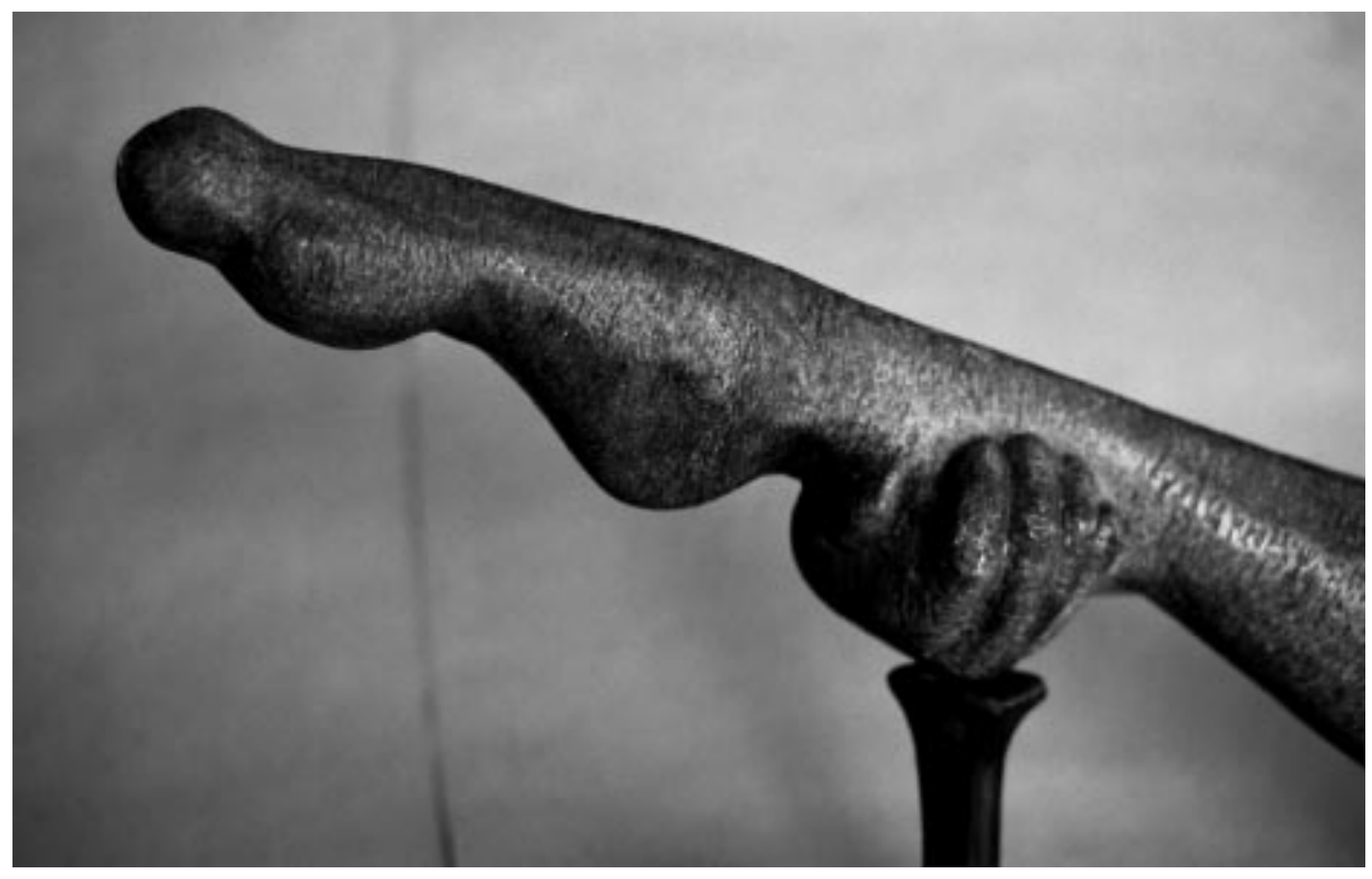

Héphaïstos le boiteux (détail), 1994. Pierre de Saint-Marc et fonte, 117 x 43 x 18cm. 


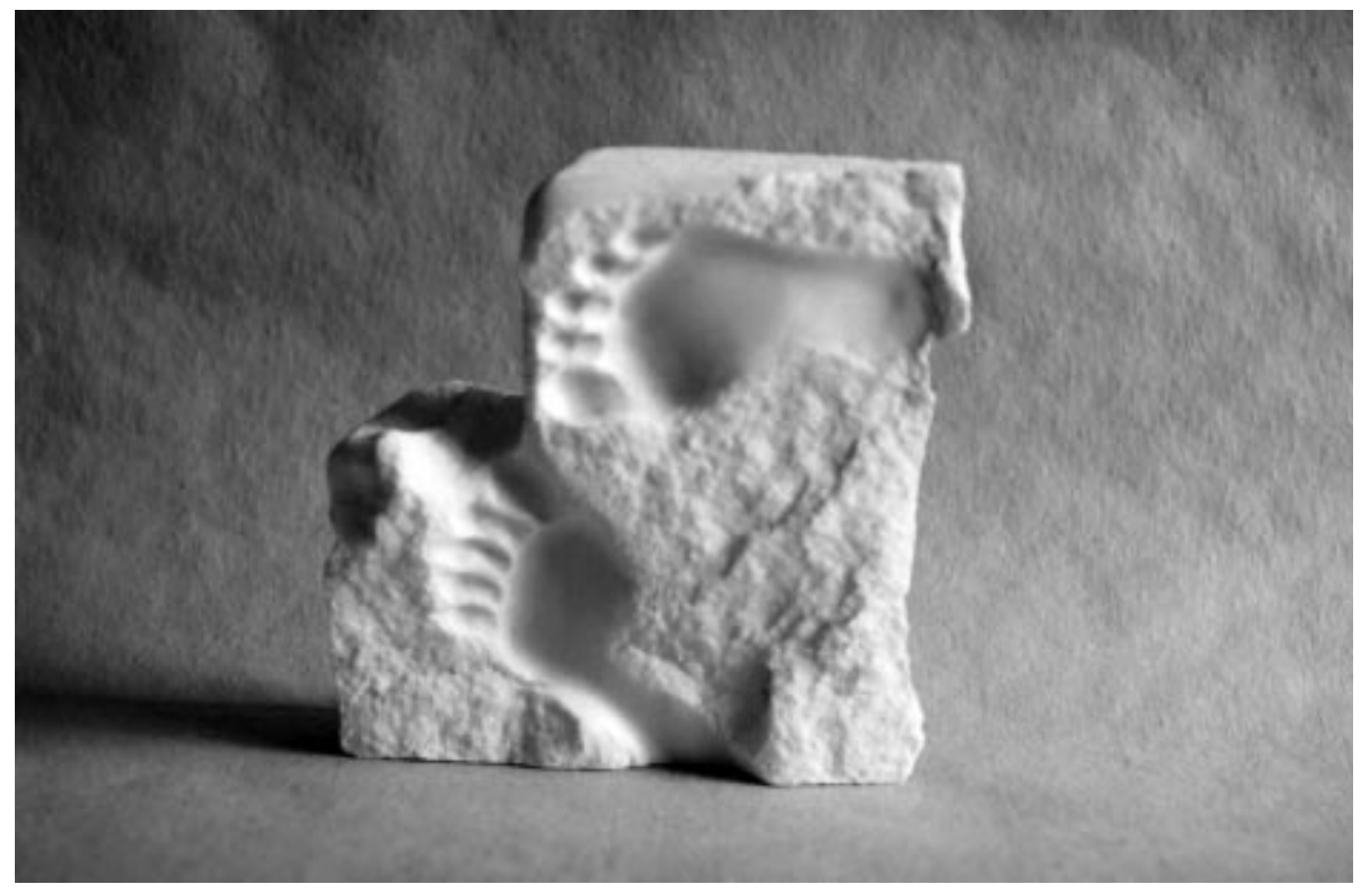

Elles sont passées (1), 2000. Marbre du Vermont, 13 × 13 × 4cm. 


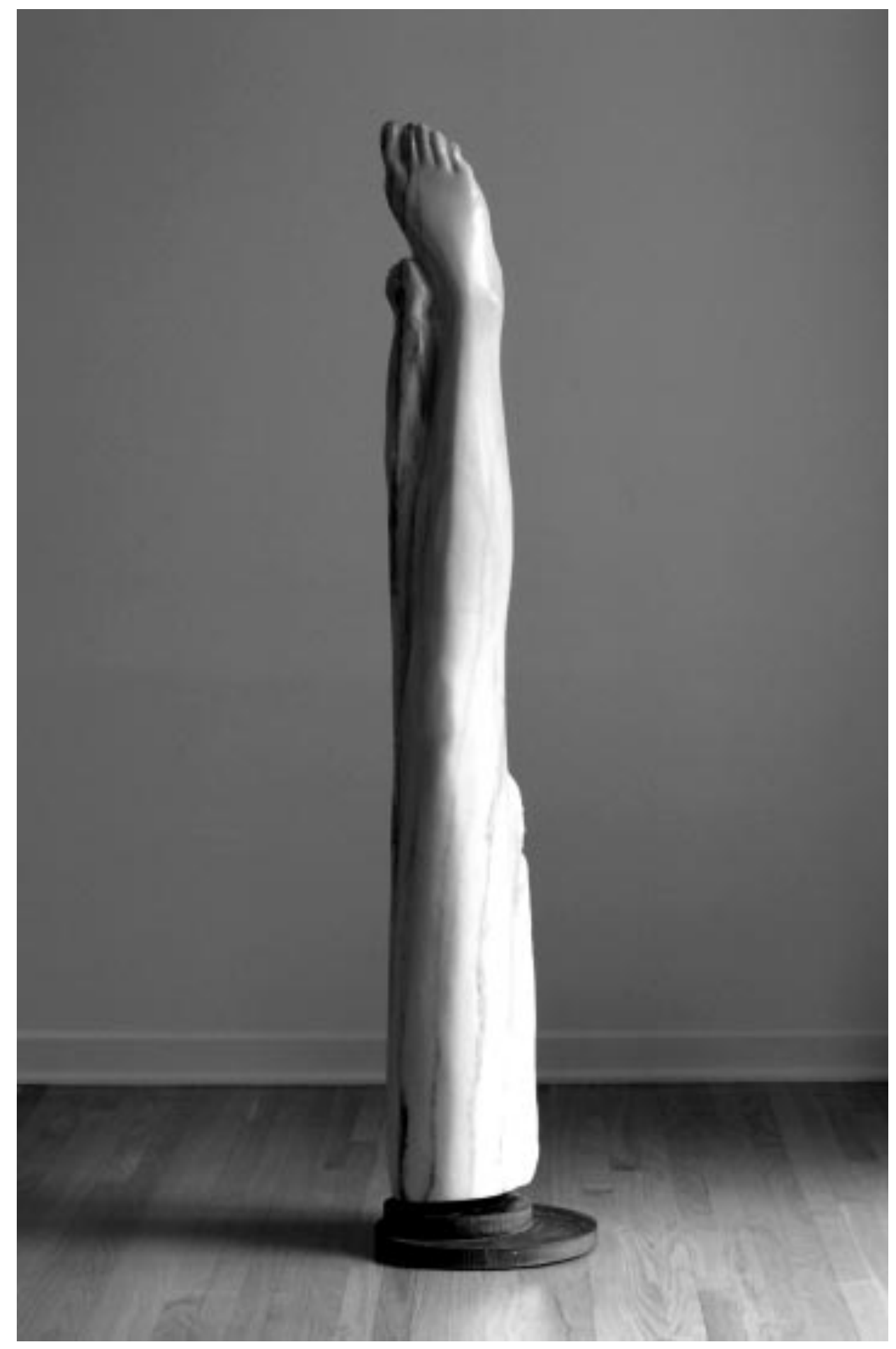

Aphrodite, 1999. Marbre du Vermont et acier, $150 \times 30 \times 18 \mathrm{~cm}$. 